Código JEL: M310

\title{
El éxito del Comercio Móvil B2C: Factores de Adopción y Propuestas de Valor de las Empresas
}

\author{
Blanca LÓPEZ-CATALÁN \\ Universidad Pablo de Olavide \\ blopcat@upo.es \\ Sonia SAN MARTÍN \\ Universidad de Burgos \\ sanmargu@ubu.es \\ Nadia JIMÉNEZ \\ Universidad de Burgos \\ nhjimenez@ubu.es
}

Recibido: 02-07-2016

Aceptado: 31-10-2016

\section{RESUMEN}

La literatura ha analizado ampliamente los factores que afectan a la adopción del comercio móvil (CM) por parte de los consumidores. Sin embargo, no ha abordado de la misma manera las propuestas de valor que el empresario puede incorporar a su oferta comercial, derivadas de las características propias del canal. Para ello, este trabajo resulta original al desarrollar una visión integradora que aborda la relación B2C en el comercio móvil. En esta línea, el objetivo de este trabajo es definir propuestas de valor basadas en la tecnología móvil incluyendo los factores involucrados en el éxito del comercio móvil desde el punto de vista del consumidor. Para conseguir este objetivo, se utiliza información secundaria y se hace una extensa revisión de la literatura que permite hacer una clasificación de las diferentes propuestas de valor sugeridas en la literatura. El estudio muestra una recopilación de los factores que favorecen la adopción del comercio móvil desde el punto de vista del consumidor, y los relaciona con seis tipos de proposiciones de valor sobre las que las empresas interesadas en la venta por móvil pueden articular su negocio para lograr que el consumidor adopte este tipo de compra. 
Palabras clave: Comercio móvil, proposiciones de valor, adopción, servicios móviles, modelos de negocio.

\title{
The Success of B2C Mobile Commerce: Adoption factors and Value propositions for Companies
}

\begin{abstract}
The literature has extensively analysed the factors affecting the adoption of this shopping channel by consumers. However, literature has not deepened in the same way on the value propositions that the firm can incorporate into its offerings including mobile phone characteristics as a channel. In order to contribute to marketing literature, we have developed a comprehensive view that addresses the $\mathrm{B} 2 \mathrm{C}$ relationship in mobile commerce. In this line, the aim of this study is to determine the value propositions based on mobile technology including the factors involved in the success of mobile commerce from the consumer 's point of view. To achieve this purpose, we used secondary information and did an extensive review of the literature to classify different proposition of value in the mobile commerce literature. As a result, this study includes several factors that favour the adoption of mobile commerce from the point of view of consumers and put a special emphasis on the offering of six types of value propositions on which companies interested in mobile selling can articulate their business to make consumers adopt this type of purchase finally.
\end{abstract}

Keywords: Mobile commerce, value propositions, adoption, mobile services, business models.

Sumario: 1. Introducción, 2. B2C m-commerce: consumidor, 3. B2C m-commerce: negocios, 4. Consideraciones 5. Referencias

\section{INTRODUCCIÓN}

La tecnología móvil está experimentando un gran avance tanto en posibilidades técnicas como en la expansión de nuevos modelos de negocio. Hasta hace muy poco tiempo el ordenador era la única posibilidad de acceso a una ingente cantidad de contenidos disponibles en Internet, que la telefonía móvil no podía asumir. Sin embargo, dos fenómenos fundamentales provocaron la aparición de un nuevo mercado: la rápida penetración de los Smartphone, cuyas características en cuanto a interactividad, inmediatez, excesiva personalización, ubicuidad, localización y conveniencia (Pedersen, 2005; Zhang et al, 2013) ofrecen un gran abanico de posibilidades tanto para empresas como para los potenciales clientes; y el abaratamiento de las tarifas de datos que ha favorecido la expansión de la 
contratación de servicios de banda ancha móvil [según la ITU (2015) en 2014 el $32 \%$ de los abonados a nivel mundial y el $83,7 \%$ de los países desarrollados]. Como consecuencia, el teléfono móvil se configura como un canal de marketing y ventas colmado de expectativas. Por lo que se refiere al posicionamiento de la empresa española, los datos de la ONTSI (2015) señalan que la banda ancha fija o móvil tiene una penetración del $98,1 \%$ entre las empresas españolas. Por otra parte, según la ONTSI (2014) el 25,6\% de los usuarios de móvil declaran haber realizado sus compras a través del móvil. En resumen, los datos reflejan una disposición favorable de los españoles hacia el comercio móvil, entendido como el proceso de compra, venta, o intercambio de productos, servicios o información a través de redes móviles con el objetivo de realizar transacciones monetarias (Turban et al, 2002; Wu y Wang, 2005). Esta posición privilegiada constituye una oportunidad real de negocio que las empresas pueden aprovechar si son capaces de comunicar propuestas de valor atractivas para los potenciales clientes.

El comercio móvil como campo de investigación está adquiriendo especial relevancia. Sin embargo, la mayor parte de la literatura se ha centrado en los factores que afectan a la adopción del comercio electrónico por parte de los potenciales clientes (Zarmpou et al., 2012). Es por ello que el objetivo de este trabajo es definir las propuestas de valor que las empresas que deseen realizar comercio móvil pueden utilizar como base para sus modelos de negocio incluyendo los factores involucrados en el éxito del comercio móvil desde el punto de vista del consumidor. Para ello, en el primer apartado del trabajo se realiza una revisión de los factores que, según la literatura, afectan a la decisión de adoptar el comercio móvil por parte de los consumidores. Posteriormente completamos el estudio desde el punto de vista de las empresas, examinando y clasificando las propuestas de valor enumeradas en anteriores estudios con el objetivo avanzar en el diseño de modelos de negocio que favorezcan el éxito del comercio móvil. De este modo, unimos la parte de la "oferta" (la parte empresarial o "B") con la parte de la "demanda" (la parte compradora o "C") para lograr una mejor comprensión de la relación de comercio móvil $\mathrm{B} 2 \mathrm{C}$. No nos consta ningún trabajo que haya intentado este esfuerzo de síntesis y de vinculación para la relación B2C en el campo del comercio móvil.

\section{EL COMERCIO MÓVIL B2C: LA "C" (CONSUMER). MOTIVOS Y BARRERAS EN DE ADOPCIÓN DEL COMERCIO MÓVIL POR LOS CONSUMIDORES}

La adopción del comercio móvil entre los particulares es una temática ampliamente analizada en la literatura, siendo el Modelo de Aceptación Tecnológica de Davis (1989), junto con la Teoría de la Acción Razonada (Fishbein y Ajzen, 1980), la Teoría del Comportamiento Planificado (Ajzen, 
1985) y la Teoría de la Difusión de las innovaciones (Rogers, 1995) las más frecuentemente utilizadas. Según estas teorías la actitud hacia la compra por móvil, la facilidad de uso, la utilidad percibida, la norma subjetiva, la compatibilidad con los valores y experiencias previas, el control percibido en la compra, las condiciones facilitadoras, las ventajas relativas de la innovación respecto a su predecesora, la posibilidad de experimentar y observar los beneficios de la compra por móvil antes de adquirir el producto o servicio o la complejidad explican por qué los usuarios de dispositivos móviles deciden adoptar esta tecnología para realizar compras.

Adicionalmente, otras teorías procedentes de la sociología, la psicología social o el marketing se han interesado por entender el fenómeno de la adopción de la compra por móvil utilizando para ello la Teoría de los usos y las gratificaciones (Katz et al., 1974) o la teoría de la Confirmación de las Expectativas (Oliver, 1980), entre otras. Dichas teorías explican el comportamiento del consumidor basado en las expectativas, el desempeño percibido, la satisfacción de la compra, el entretenimiento y disfrute percibidos, el estatus, la afinidad con el medio móvil o incluso la capacidad de expresarse a través de este medio visto desde el punto de vista psico-sociológico.

En forma de síntesis, podemos indicar que los factores recogidos en la literatura sobre el punto de vista del consumidor en lo que a adopción de compra por móvil se refiere, pueden agruparse en tres categorías atendiendo a la naturaleza de los aspectos a los que hacen referencia, como se refleja en el cuadro 1: factores con componente tecnológico o relacionadas con el campo de sistemas de información, factores de naturaleza psico-sociológica y factores relativos al marketing, tanto desde el punto de vista relacional como del valor percibido aplicado al comercio móvil. El cuadro 1 muestra dichos factores como variables que afectan a la adopción de la compra con teléfono móvil, junto con la descripción de los conceptos y referencias en la literatura de comercio móvil. 
Cuadro 1. Revisión y clasificación de factores de la literatura de adopción de compra por móvil.

\begin{tabular}{|c|c|c|}
\hline \multicolumn{3}{|c|}{ FACTORES DE COMPONENTE TECNOLÓGICO } \\
\hline FACTOR & REFERENCIAS & CONCEPTO \\
\hline Calidad del sistema & $\begin{array}{l}\text { Gu et al., (2009); Chemingui } \\
\text { y Ben lallouna (2013) }\end{array}$ & Velocidad de la red y estabilidad del sistema \\
\hline Compatibilidad & $\begin{array}{l}\text { Chen (2008); Khraim et al. } \\
\text { (2011); Chemingui y Ben } \\
\text { lallouna (2013) }\end{array}$ & $\begin{array}{l}\text { Grado por el cual, realizar transacciones de comercio móvil } \\
\text { se percibe como consistente con los valores, necesidades y } \\
\text { experiencias pasadas de los potenciales clientes. }\end{array}$ \\
\hline $\begin{array}{l}\text { Complejidad de los } \\
\text { medios de pago }\end{array}$ & $\begin{array}{l}\text { Mallat (2007); Khraim et al. } \\
\qquad(2011)\end{array}$ & $\begin{array}{l}\text { Grado por el cual un medio de pago es percibido como } \\
\text { complicado de usar y entender }\end{array}$ \\
\hline $\begin{array}{l}\text { Condiciones } \\
\text { facilitadoras }\end{array}$ & $\begin{array}{l}\mathrm{Lu}(2005) \text {; Chemingui y Ben } \\
\text { lallouna (2013) }\end{array}$ & $\begin{array}{l}\text { Grado y tipo de soporte ofrecido a un individuo en el uso } \\
\text { de una tecnología. }\end{array}$ \\
\hline $\begin{array}{l}\text { Confiabilidad del } \\
\text { sistema }\end{array}$ & $\begin{array}{l}\text { Mallat et al. (2008); Khalifa } \\
\text { et al. (2008) }\end{array}$ & Confianza con el entorno tecnológico \\
\hline Eficiencia & Khalifa et al. (2008) & Navegación y procesos de transacción eficientes \\
\hline $\begin{array}{l}\text { Facilidad de uso } \\
\text { percibida }\end{array}$ & $\begin{array}{l}\text { Wu et al. (2010); Sripalawat } \\
\text { t al. (2011); Agrebi y Jallais } \\
\text { (2015); Liébana-Cabanillas } \\
\text { et al. (2014); Thakur (2014) }\end{array}$ & $\begin{array}{l}\text { Grado en el que una persona piensa que utilizar una } \\
\text { determinada aplicación estará exento de esfuerzo. }\end{array}$ \\
\hline Interactividad & Koivumäki et al. (2008) & Diálogo entre un sitio y sus visitantes \\
\hline Movilidad & Mallat et al. (2008) & Beneficios por tiempo, lugar, acceso a servicios y uso \\
\hline $\begin{array}{l}\text { Posibilidad de } \\
\text { experimentar } \\
\text { previamente con la } \\
\text { tecnología } \\
\end{array}$ & $\begin{array}{l}\text { Mallat et al. (2008); Khraim } \\
\text { et al. (2011); Chemingui y } \\
\text { Ben lallouna (2013) }\end{array}$ & $\begin{array}{l}\text { Grado en que el potencial usuario percibe que el medio de } \\
\text { pago móvil aumenta la conveniencia en el proceso de pago }\end{array}$ \\
\hline Utilidad percibida & $\begin{array}{l}\text { Wu et al. (2005); Sripalawat } \\
\text { et al. (2011); Agrebi y Jallais } \\
\text { (2015); Liébana-Cabanillas } \\
\text { et al. (2014) }\end{array}$ & $\begin{array}{l}\text { Grado por el cual un individuo piensa que la utilización de } \\
\text { una determinada aplicación mejorará su rendimiento. }\end{array}$ \\
\hline $\begin{array}{l}\text { Velocidad de } \\
\text { transacción }\end{array}$ & Chen (2008) & $\begin{array}{l}\text { Grado en que el potencial usuario percibe que el medio de } \\
\text { pago móvil aumenta la velocidad de la transacción. }\end{array}$ \\
\hline Ventaja relativa & $\begin{array}{l}\text { Mallat (2007); Khraim et al. } \\
\qquad(2011)\end{array}$ & $\begin{array}{l}\text { Grado en el que una innovación se percibe como mejor que } \\
\text { su precursora. }\end{array}$ \\
\hline \multicolumn{3}{|c|}{ FACTORES DE COMPONENTE PSICO-SOCIAL } \\
\hline $\begin{array}{l}\text { Afinidad con el uso del } \\
\text { teléfono móvil }\end{array}$ & $\begin{array}{l}\text { Bigné et al. (2007); Liu } \\
\text { et al. (2015) }\end{array}$ & La importancia de un medio en la vida de la persona. \\
\hline Ansiedad & $\mathrm{Lu} \mathrm{y} \mathrm{Su} \mathrm{(2009)}$ & Emociones negativas en estados cognitivos \\
\hline Autoeficacia & $\begin{array}{l}\text { Sripalawat et al. } \\
(2011) ; \text { Khraim et al. } \\
(2011)\end{array}$ & $\begin{array}{l}\text { Confianza que un individuo tiene sobre su capacidad de } \\
\text { desarrollar un determinado comportamiento }\end{array}$ \\
\hline Control percibido & $\begin{array}{l}\text { Bhattti (2007); Song et } \\
\text { al. (2015) }\end{array}$ & $\begin{array}{l}\text { Percepción de un individuo sobre lo fácil o difícil que } \\
\text { puede ser realizar un comportamiento determinado. }\end{array}$ \\
\hline $\begin{array}{c}\text { Estatus/pertenencia a clas } \\
\text { social }\end{array}$ & $\begin{array}{l}\text { (Hsu et al. (2007); } \\
\text { Liébana-Cabanillas et } \\
\text { al. (2014) }\end{array}$ & $\begin{array}{l}\text { Grado en el que un individuo percibe que el uso de una } \\
\text { innovación mejora su propia imagen o estatus. }\end{array}$ \\
\hline
\end{tabular}




\begin{tabular}{|c|c|c|}
\hline Normas subjetivas & $\begin{array}{c}\text { Pedersen E. (2005); Gu } \\
\text { et al. (2009); } \\
\text { Sripalawat et al. } \\
\text { (2011); Liébana- } \\
\text { Cabanillas et al. (2014) }\end{array}$ & $\begin{array}{l}\text { Percepción de un individuo de que la mayoría de las } \\
\text { personas importantes para él creen que debería o no debería } \\
\text { realizar un determinado comportamiento en cuestión. }\end{array}$ \\
\hline \multicolumn{3}{|c|}{ FACTORES DE MARKETING } \\
\hline FACTOR & REFERENCIAS & CONCEPTO \\
\hline Calidad percibida & $\begin{array}{l}\text { Turel (2007); Kim et al. } \\
\text { (2007) }\end{array}$ & Estabilidad y eficiencia percibida de un sistema \\
\hline Atención al cliente & Thakur (2014) & $\begin{array}{l}\text { Servicio proporcionado a los clientes durante situaciones } \\
\text { problemáticas a través de centros de atención que implican } \\
\text { el uso de tecnología móvil. }\end{array}$ \\
\hline Confianza & $\begin{array}{c}\text { Mallat (2007); Gu et } \\
\text { al., (2009); Davis } \\
\text { (2011); Liébana- } \\
\text { Cabanillas et al. (2014); } \\
\text { Thakur (2014); } \\
\text { Chemingui y Ben } \\
\text { lallouna (2013) }\end{array}$ & $\begin{array}{l}\text { Grado en que el potencial usuario está dispuesto a asumir } \\
\text { vulnerabilidad en una relación. }\end{array}$ \\
\hline Conveniencia & Khalifa y Shen (2008) & $\begin{array}{l}\text { Grado en el que el comercio móvil hace más fácil para los } \\
\text { clientes realizar transacciones }\end{array}$ \\
\hline Coste-precio & $\begin{array}{l}\text { Mallat et al. (2008); } \\
\text { Sripalawat et al. (2011) }\end{array}$ & $\begin{array}{l}\text { Posibles gastos derivados del comercio móvil, como coste } \\
\text { de equipo, acceso o cuotas. }\end{array}$ \\
\hline Disfrute /placer percibidos & $\begin{array}{l}\text { Ha et al. (2007); Mallat } \\
\text { et al. (2008); Agrebi y } \\
\text { Jallais (2015); } \\
\text { Chemingui y Ben } \\
\text { lallouna (2013) }\end{array}$ & $\begin{array}{l}\text { Grado en el que el comercio móvil es percibido como } \\
\text { divertido independientemente del rendimiento obtenido. }\end{array}$ \\
\hline $\begin{array}{l}\text { Facilidad de observar o } \\
\text { descubrir los beneficios } \\
\text { tangibles o intangibles del } \\
\text { producto }\end{array}$ & $\begin{array}{l}\text { Hsu et al. (2007); Chen } \\
\text { et al. (2009) }\end{array}$ & $\begin{array}{l}\text { Facilidad para observar o descubrir los beneficios tangibles } \\
\text { o intangibles del producto }\end{array}$ \\
\hline $\begin{array}{l}\text { Posibilidad de } \\
\text { experimentar previamente } \\
\text { con el producto }\end{array}$ & $\begin{array}{l}\text { Chen et al. (2009); } \\
\text { Khraim et al. (2011) }\end{array}$ & El nuevo producto permite ser probado por no expertos \\
\hline Riesgo percibido & $\begin{array}{c}\text { Mallat, (2007); } \\
\text { Sripalawat et al. } \\
\text { (2011); Liébana- } \\
\text { Cabanillas et al. (2014); } \\
\text { Yang et al. (2015) }\end{array}$ & $\begin{array}{l}\text { Expectativa subjetiva del usuario de sufrir una pérdida en } \\
\text { detrimento de los resultados deseados al realizar comercio } \\
\text { móvil. }\end{array}$ \\
\hline Satisfacción & $\begin{array}{l}\text { Püschel et al. (2010); } \\
\text { Agrebi y Jallais (2015); } \\
\text { Thakur (2014) }\end{array}$ & $\begin{array}{l}\text { El nivel de conformidad tras comparar el rendimiento } \\
\text { percibido de un producto con las expectativas del } \\
\text { consumidor. }\end{array}$ \\
\hline
\end{tabular}

Fuente: Elaboración propia

Como se desprende del cuadro anterior, muchas son las variables facilitadoras de la adopción. Sin embargo, por sí mismas no son suficientes para estimular la compra por móvil. ¿Qué beneficios deben ofrecer las empresas para facilitar las compras? En el caso del comercio móvil, el éxito de la oferta comercial pasa por incorporar en la propuesta de valor ventajas inherentes al nuevo canal. 


\section{EL COMERCIO MÓVIL B2C: LA "B" (BUSINESS)}

Con el fin de responder a los motivos que pueden conducir al consumidor a la adopción del comercio móvil, la empresa tiene a su alcance diferentes proposiciones de valor que puede utilizar. La proposición de valor es un concepto clásico de marketing que relaciona la oferta y las necesidades del cliente (Porter, 1998). Según Anderson (2006), los proveedores de bienes y/o servicios usan el término "propuesta de valor" de tres maneras diferentes: simplemente enumerando los beneficios que su oferta de bienes o servicios puede proporcionar al cliente; centrándose en los aspectos favorables que diferencian su oferta de la de la siguiente mejor alternativa con la suposición que estas diferencias brindan valor a sus clientes; y enfocándose en la investigación, con el objetivo de entender qué elementos son los más importantes para el mercado objetivo. El valor que se deriva de estas aproximaciones, no sólo está influenciado por el uso de los productos o servicios, sino también por la utilización del canal, ya que los clientes evalúan el valor del canal móvil comparado con otras alternativas (Anckar y D'Incau 2002). En consecuencia, es necesario definir las características del nuevo canal que son susceptibles de formar parte de las propuestas de valor definidas por las empresas, y ponerlas en relación con los factores que favorecen la adopción de la compra por parte del cliente. Puesto que las propuestas de valor en el comercio móvil se derivan fundamentalmente de las condiciones de movilidad (Yuang y Zang, 2003; Groß, 2015), las hemos clasificado en seis grandes bloques representados en el cuadro 2. En él se especifican aspectos detallados por los distintos autores, con el objetivo de individualizar y diferenciar la propuesta de valor de cada producto o servicio en base a los bloques descritos.

El primer bloque se refiere a las propuestas de valor relacionadas con el espacio, que Balasubraman et al. (2002) describen como dimensiones que reflejan cierta "sensibilidad a la localización", identificado por Mallat et al. (2008) como factor de adopción. En este sentido Clarke (2001) y Zhang et al. (2013) diferencian entre la ubicuidad, relativo a la posibilidad de recibir información, producirla o realizar transacciones desde cualquier lugar; y la localización, que hace mención a la posibilidad de conocer la situación geográfica del dispositivo móvil, lo cual le hace susceptible de recibir información contextualizada en base a dicha localización. Es el caso del marketing de proximidad, que permite el envío y recepción de información adaptada a la situación geográfica del terminal.

El segundo bloque incluye dos propuestas de valor relativas a la relación comercial: la personalización y la interactividad. La personalización e identificación a través de la identidad del módulo suscriptor o tarjeta SIM (Lee y Ho, 2010; Zhang et al., 2013) hace referencia al carácter individual y único de los dispositivos móviles, que facilitan la adaptación de la oferta al cliente convirtiéndole en un objetivo de marketing one to one a través de distintas estrategias de marketing móvil. Por otra parte, Koivumäki et al. (2008) 
demostraron que la capacidad de diálogo entre un sitio y sus visitantes afecta positivamente a la adopción en la compra. La denominada interactividad (Pedersen, 2005; Turban et al, 2002) manifiesta la posibilidad de interacción de forma directa y personal entre empresa y consumidor. Refleja un valor esencial en modelos de negocio de éxito donde la capacidad de relación mutua es clave, ya que favorece la aparición y el mantenimiento de la confianza (Davis, 2011; Hung et al., 2012; Jayawardhena et al., 2009), y la satisfacción (Püshel et al, 2010; Hung et al., 2012), variables esenciales en la literatura de adopción del comercio móvil. También ayudan a mejorar en el control percibido (Bhatti, 2007; Jayawardhena et al., 2009), la seguridad y a disminuir el riesgo percibido (Sripalawat et al, 2011; Wu y Wang, 2006) y las emociones negativas, como la ansiedad (Lu y Su, 2009; Li et al., 2012) favoreciendo la compra a través del dispositivo móvil.

El tercer bloque de propuestas de valor está relacionado con el momento de la compra. En este grupo de propuestas, la aportación de valor se deriva de que la adquisición de bienes y servicios es sensible a la variable tiempo (Balasubraman et al, 2002). En este sentido, la posibilidad de acceder a servicios de urgencia y de cubrir necesidades espontáneas no programadas se han señalado como aportaciones de valor del comercio móvil (Anckar y D’Incacau, 2002). Aunque estas propuestas esconden tras de sí la movilidad, el énfasis se sitúa en el factor temporal. Atendiendo a Zhang et al. (2013), el móvil proporciona a los usuarios una conectividad permanente e instantánea con la empresa. En este sentido, la eficiencia del comercio móvil en cuanto a ahorro de tiempo para el cliente, se presenta en la literatura como un beneficio especialmente valorado (Yuang y Zhang, 2003; Laukkanen y Pasanen, 2008). Finalmente, Lee y Ho (2010) afirman que uno de los factores de éxito que permite a las empresas que realizan comercio móvil crear ventajas competitivas sostenibles es la accesibilidad, entendida como la capacidad de contactar con el usuario en cualquier momento (como ocurre en los sistemas de alertas, entre otros).

Un cuarto bloque de propuestas engloba los atributos del producto/servicio comprado. Para Thorbjørnsen et al. (2007) el valor que un usuario atribuye a un servicio determinado viene determinado por los atributos intrínsecos del servicio, que están presentes en muchas de las variables estudiadas desde el punto de vista de la adopción de la tecnología, como la facilidad de uso (Wu et al, 2010; Agrebi y Jallais, 2015) y la utilidad percibida (Sripalawat et al, 2011; Liébana-Cabanillas et al., 2014). En este contexto, también la posibilidad de experimentar con el producto y con la tecnología antes de la compra, o la facilidad para descubrir los beneficios del producto o servicio favorecen la adopción del mismo (Chen et al, 2009; Kraim et al, 2011; Agrebi y Jallais, 2015). Por este motivo, el grado de soporte ofrecido en el uso de esta tecnología durante la compra (condiciones facilitadoras) es valorado por los clientes, según demuestra Lu (2005) y Chemingui y Ben lallouna (2013). Los anteriores atributos mejoran la calidad percibida (Turel et al, 2007; Thakur, 2014) y pueden favorecer la percepción de una ventaja relativa respecto a 
otras formas de compra (Mallat, 2007; Groß, 2015) como el comercio electrónico tradicional. En relación a los atributos del servicio, Park et al. (2011) señalan como aportación de valor la posibilidad de cubrir las necesidades de entretenimiento independientemente del rendimiento obtenido. El también denominado disfrute percibido, se ha demostrado un componente clave en la adopción del comercio móvil (Mallat et al, 2008; Agrebi y Jallais, 2015), por lo que su inclusión en la proposición de valor resulta beneficiosa. Finalmente, las utilidades personales relacionadas con los pagos, denominadas genéricamente "monedero de bolsillo" son presentadas como fuente de ventajas competitivas para la empresa según Mas y Radcliffe (2011). Sin embargo, es necesario considerar que la complejidad de los medios de pago supone un freno a la adopción en el comercio móvil (Mallat, 2007), así como los gastos asociados a este tipo de compra (Sripalawat et al, 2001) por lo que sería conveniente que las empresas potenciaran la autoeficacia (Khraim et al, 2011) y la conveniencia (Khalifa y Shen, 2008), definida esta última como la agilidad y accesibilidad que proporciona el comercio móvil y que se traduce en una mejora de la calidad de vida de los usuarios (Pedersen, 2005) y, un determinante de la continuidad de comercio móvil (Zhou, 2014). Asimismo, trasladar al consumidor señales sobre la velocidad de la transacción referida a los medios de pago facilitaría la adopción, según Chen, (2008).

En un contexto como el comercio móvil las percepciones del cliente sobre el entorno tecnológico son clave. Por ello, el quinto bloque, sugerido por Thorbjørnsen et al. (2007), plantea como propuestas de valor los atributos del sistema. La confianza en el entorno tecnológico, también denominado confiabilidad del sistema, fue identificado por Khalifa et al. (2008) como factores favorecedores de la adopción tecnológica junto con la percepción de eficiencia en la navegación y en los procesos durante la compra. Asimismo, Gu et al. (2009) demostraron que la estabilidad del sistema influye positivamente en el uso de la banca móvil. Estos aspectos, íntimamente ligados a la naturaleza del canal, afectan directamente a la confianza y, por tanto, son grandes candidatos a formar parte de las propuestas de valor definidas por las empresas, especialmente en servicios financieros o relacionados con transacciones de alto nivel de implicación. Los atributos complementarios a la red hacen referencia a características que ofrecen soporte a aplicaciones o servicios ya existentes (Constantinescu et al., 2014). Es decir, aportan complementariedad a otros servicios, como ocurre en las plataformas que sirven de repositorio a aplicaciones móviles, por ejemplo.

El sexto bloque refleja la comunicación comercial con la empresa como fuente de ventajas competitivas, que se concreta en la publicidad y el marketing de permiso como aportación de valor para los clientes. La tecnología móvil constituye en nuestros días un hecho social. La utilización de dispositivos móviles en las compras es percibida por los individuos como un signo de estatus (Hsu et al, 2007), y la influencia del grupo en la decisión de adoptar este canal está ampliamente contrastada (Gu et al, 2009; Lu, 2014). Por ello, los proveedores de servicios 
móviles luchan por destacar con la publicidad la idea que la utilización del dispositivo móvil es consistente con los valores y necesidades de los potenciales clientes (Chen, 2008), buscando representar la afinidad del dispositivo con la vida de los potenciales clientes (Bigné et al, 2007). En este sentido, es interesante resaltar las ventajas que reporta a ambas partes de la relación el marketing de permiso y la integración del comercio móvil en el CRM de la empresa (el denominado Mobile-Crm) (Awasthi y Sangle, 2012).

Cuadro 2. Revisión y clasificación de propuestas de valor sugeridas en la literatura de comercio móvil.

\begin{tabular}{|c|c|c|}
\hline $\begin{array}{c}\text { PROPUESTA DE } \\
\text { VALOR }\end{array}$ & $\begin{array}{c}\text { SELECCIÓN DE } \\
\text { AUTORES }\end{array}$ & $\begin{array}{l}\text { DESCRIPCIÓN DE LA } \\
\text { PROPUESTA DE VALOR } \\
\text { PLANTEADA }\end{array}$ \\
\hline \multicolumn{3}{|c|}{ 1. REFERENTES AL ESPACIO } \\
\hline Ubicuidad & $\begin{array}{l}\text { Balasubraman et al. } \\
\text { (2002) Clarke (2001); } \\
\text { Yun et al. (2011); Chan y } \\
\text { Yee-Loong (2013) }\end{array}$ & $\begin{array}{l}\text { Posibilidad de recibir información, } \\
\text { producirla o realizar transacciones desde } \\
\text { cualquier localización e } \\
\text { independientemente del horario. }\end{array}$ \\
\hline Localización & $\begin{array}{l}\text { Clarke (2001); Pedersen } \\
\text { (2005); Lee y Ho(2010); } \\
\text { Chan y Yee-Loong } \\
\text { (2013); Groß, }(2015)\end{array}$ & $\begin{array}{l}\text { Referido a la posibilidad de conocer la } \\
\text { localización del dispositivo móvil, lo } \\
\text { cual le hace susceptible de recibir } \\
\text { información contextualizada en base a la } \\
\text { localización. }\end{array}$ \\
\hline \multicolumn{3}{|c|}{ 2. REFERENTES AL SERVICIO/RELACIÓN: } \\
\hline Conveniencia & $\begin{array}{l}\text { Pedersen (2005); Lee y } \\
\text { Ho (2010); Nasri (2011); } \\
\text { Groß (2015) }\end{array}$ & $\begin{array}{l}\text { Agilidad y accesibilidad que } \\
\text { proporciona el comercio móvil y que } \\
\text { permite traducirse en una mejora de la } \\
\text { calidad de vida de los usuarios }\end{array}$ \\
\hline Personalización & $\begin{array}{l}\text { Clarke (2001); Lee y Ho } \\
\text { (2010); Chan y Yee- } \\
\text { Loong }(2013)\end{array}$ & $\begin{array}{l}\text { Los dispositivos móviles se emplean } \\
\text { habitualmente por un único usuario, lo } \\
\text { que posibilita que los servicios } \\
\text { suministrados se adapten a las } \\
\text { necesidades del usuario. }\end{array}$ \\
\hline Interactividad & $\begin{array}{c}\text { Turban et al. (2002); } \\
\text { Pedersen (2005); Kang y } \\
\text { Johnson (2014) }\end{array}$ & $\begin{array}{l}\text { Posibilidad de interacción de forma } \\
\text { directa y personal entre empresa y } \\
\text { consumidor. }\end{array}$ \\
\hline \multicolumn{3}{|c|}{ 3. REFERENTES AL MOMENTO DE LA COMPRA } \\
\hline Urgencia & $\begin{array}{l}\text { Anckar y D'Íncau (2002); } \\
\text { Balasubraman et al. } \\
\text { (2002); Yuang y Zhang } \\
\text { (2003); Chemingui y Ben } \\
\text { lallouna (2013) }\end{array}$ & $\begin{array}{l}\text { Posibilidad de cubrir necesidades } \\
\text { caracterizadas por la urgencia debido a } \\
\text { factores externos o posibilidad de } \\
\text { acceder a ciertos servicios donde el } \\
\text { tiempo es el factor crítico. }\end{array}$ \\
\hline
\end{tabular}




\begin{tabular}{|c|c|c|}
\hline Espontaneidad & $\begin{array}{l}\text { Anckar y D'Íncau (2002); } \\
\text { Jahanshahi et al. (2011) }\end{array}$ & $\begin{array}{l}\text { Posibilidad de satisfacer necesidades } \\
\text { que se generan de forma espontánea, no } \\
\text { como consecuencia directa de un factor } \\
\text { externo. Incluye las compras por } \\
\text { impulso. }\end{array}$ \\
\hline Eficiencia & $\begin{array}{l}\text { Anckar y D'Íncau (2002); } \\
\text { Yuang y Zhang (2003); } \\
\text { Jahanshahi et al. (2011) }\end{array}$ & $\begin{array}{l}\text { Posibilidad de incrementar la } \\
\text { productividad y eficiencia de tareas } \\
\text { cotidianas, al facilitar gran ahorro de } \\
\text { tiempo. }\end{array}$ \\
\hline Accesibilidad & $\begin{array}{l}\text { Lee y Ho (2010); Kang y } \\
\text { Johnson (2014); Agrebi y } \\
\text { Jallais (2015) }\end{array}$ & $\begin{array}{l}\text { La posibilidad de que el usuario sea } \\
\text { contactado en cualquier momento puede } \\
\text { ser un criterio valorado por el } \\
\text { consumidor. }\end{array}$ \\
\hline \multicolumn{3}{|c|}{$\begin{array}{l}\text { 4. REFERENTES A LOS ATRIBUTOS DEL PRODUCTO/SERVICIO } \\
\text { COMPRADO: }\end{array}$} \\
\hline $\begin{array}{l}\text { Monedero de } \\
\text { bolsillo }\end{array}$ & $\begin{array}{l}\text { Valsecchi et al. (2007); } \\
\text { Mas y Radcliffe (2011) }\end{array}$ & $\begin{array}{l}\text { Utilidades personales relacionadas con } \\
\text { los pagos }\end{array}$ \\
\hline $\begin{array}{l}\text { Atributos } \\
\text { intrínsecos del } \\
\text { servicio }\end{array}$ & $\begin{array}{l}\text { Thorbjørnsen et al. } \\
\text { (2007); Chemingui y Ben } \\
\text { lallouna (2013) }\end{array}$ & $\begin{array}{l}\text { Características intrínsecas del } \\
\text { producto/servicio. }\end{array}$ \\
\hline Entretenimiento & $\begin{array}{l}\text { Park et al. (2011); Agrebi } \\
\text { y Jallais (2015) }\end{array}$ & $\begin{array}{l}\text { Posibilidad de cubrir necesidades de } \\
\text { ocio independientemente del } \\
\text { rendimiento obtenido. }\end{array}$ \\
\hline \multicolumn{3}{|c|}{ 5. REFERENTES A LOS ATRIBUTOS DEL SISTEMA: } \\
\hline $\begin{array}{l}\text { Atributos de la red } \\
\text { del usuario }\end{array}$ & $\begin{array}{c}\text { Thorbjornsen et al. } \\
\text { (2007); Constantinescu et } \\
\text { al. (2014) }\end{array}$ & $\begin{array}{l}\text { El valor depende de las características } \\
\text { de la red (tamaño, rapidez, fiabilidad, } \\
\text { etc.) }\end{array}$ \\
\hline $\begin{array}{l}\text { Atributos } \\
\text { complementarios a } \\
\text { la red }\end{array}$ & $\begin{array}{l}\text { Thorbjornsen et al. } \\
\text { (2007); Constantinescu et } \\
\text { al. (2014) }\end{array}$ & $\begin{array}{l}\text { La existencia de atributos que } \\
\text { complementan las características de la } \\
\text { red, generan valor para los clientes (por } \\
\text { ejemplo, la existencia de una plataforma } \\
\text { para la gestión de los libros } \\
\text { electrónicos) }\end{array}$ \\
\hline \multicolumn{3}{|c|}{ 6. REFERENTES A LA COMUNICACIÓN COMERCIAL CON LA EMPRESA: } \\
\hline $\begin{array}{l}\text { Publicidad y } \\
\text { marketing de } \\
\text { permiso }\end{array}$ & Elaboración propia & $\begin{array}{l}\text { Aplicación de un enfoque CRM de } \\
\text { comunicación con la cartera de clientes } \\
\text { de la empresa que fomente el efecto } \\
\text { boca-oreja (WOM) y la difusión de las } \\
\text { tecnologías entre los miembros del } \\
\text { grupo social. }\end{array}$ \\
\hline
\end{tabular}

Fuente: Elaboración Propia 


\section{CONSIDERACIONES FINALES}

En entornos cambiantes como el comercio móvil, la orientación al mercado de las empresas es fundamental para alcanzar el éxito. La literatura ha prestado especial interés en conocer los factores que afectan a la adopción del comercio móvil por parte de los potenciales consumidores. Sin embargo, no existe apenas literatura que analice expresamente cuáles son las propuestas de valor relacionadas con el nuevo canal. El conocimiento de los factores de adopción resulta insuficiente para obtener el éxito empresarial. Las organizaciones necesitan identificar los motivos y las necesidades de los clientes, y además conocer las dimensiones específicas del comercio móvil para situarlas como base para la creación de valor en sus modelos de negocio, obteniendo así niveles de diferenciación. El objetivo de este trabajo es definir propuestas de valor basadas en la tecnología móvil incluyendo asimismo los factores involucrados en el éxito del comercio móvil desde el punto de vista del consumidor. Para ello hemos realizado una revisión de la literatura que identifica, por una parte, factores de adopción del comercio móvil y por otra, propuestas de valor. Como resultado presentamos seis tipos de proposiciones de valor sobre las que las empresas pueden articular los modelos de negocio para responder al comprador potencial por móvil, y las hemos relacionado con los factores que inciden en la adopción de este tipo de negocio por parte de los potenciales clientes.

La primera referente al espacio, en virtud de la cual las empresas orientarían su negocio de éxito hacia servicios móviles valorados especialmente por el consumidor que se encuentra en cualquier entorno de movilidad. También referente al espacio serían especialmente valorados, según las propuestas realizadas, servicios basados en la localización del consumidor, o georeferenciados (como servicios en rutas turísticas u eventos específicos).

El segundo tipo de propuestas hace referencia a características específicas del servicio móvil como las posibilidades de personalización y la interactividad. El comercio móvil está produciendo cambios en la forma en que los consumidores consumen productos y servicios, los consumidores son más exigentes, y buscan mayor personalización del servicio. Según la literatura, la habilitación de espacios para favorecer la interactividad entre empresas y visitantes favorece la compra mediante la creación y mantenimiento de relaciones entre proveedor de servicios y consumidor. En este sentido, las variables relacionales como la confianza o la satisfacción cobran especial relevancia. Esta propuesta de valor, estaría especialmente indicada para servicios donde sea esencial favorecer el control percibido, reduciendo la ansiedad y la percepción de riesgo.

La tercera propuesta de valor apunta a la creación de servicios móviles donde el tiempo se convierte en un factor crítico. Sin embargo, lo crítico de este factor ha de ser detallado en la propuesta. Algunos servicios móviles asisten en momentos de urgencia (por localizar servicios médicos disponibles); otros satisfacen las compras 
por impulso (como participación en concursos); y otros venden maximizar la eficacia personal (organizadores, o aplicaciones orientadas al apoyar el estilo de vida de los clientes potenciales). También la accesibilidad es identificada como valor para usuarios que deseen estar "siempre accesibles" (como aplicaciones mobile learning, entre otros). Otra propuesta de valor para potenciales modelos de negocio se basa en fomentar los atributos de los productos o servicios móviles. Hemos destacado algunos tan específicos como las aplicaciones personales relacionadas con los pagos y los atributos especialmente enfocados a generar entretenimiento. En este sentido, la inclusión de características o actividades generadoras de disfrute en el modelo de negocio, independientemente del tipo de producto o servicio, provocará un valor añadido para el cliente.

Además, hemos podido identificar que atributos intrínsecos al servicio relacionados con la adopción de innovaciones que pueden ser utilizados por las empresas para favorecer la adopción de la compra. Destacar del producto o servicio su facilidad de uso, utilidad, posibilidad de experimentación previa a la compra, y ofrecer ayuda durante el proceso facilitan la percepción de ventajas competitivas que pueden impulsar el comercio móvil. Por otra parte, todas estas variables tomadas de la literatura trabajan en pos de la calidad percibida, sin duda, factor vital en la precepción de valor.

El grupo de propuestas relativas al sistema apoyarán modelos de negocio mediante ofertas complementarias a otros existentes en la red, así como servicios que aprovechen las ventajas de los avances tecnológicos que proporciona mejoras en la red del usuario. Este grupo de propuestas entendemos que serán especialmente valoradas por usuarios avanzados tecnológicamente, que examinarán tanto la eficiencia en los procesos de compra como la estabilidad del sistema. La última propuesta de valor que planteamos es la referente a la posibilidad de establecer comunicación comercial con la empresa. El potencial comprador móvil es un cliente predispuesto a obtener de forma eficiente información relevante para su toma de decisiones de compra. Por este motivo es más probable que valore la información comercial que se le brinde, siempre que haya sido emitida con su consentimiento expreso, para lo cual es recomendable utilizar marketing de permiso. Asimismo, sería deseable que las estrategias de marketing contemplaran los aspectos conductuales identificados en la literatura de adopción. El deseo del usuario de exhibir cierto estatus, la relación de la compra con el estilo de vida y la importancia de las normas subjetivas son aspectos beneficiosos para las compras. A ese respecto es recomendable facilitar canales al cliente donde generar un efecto boca oreja (WOM) que retroalimentaría el efecto positivo de la norma subjetiva como factor de adopción. Es por ello que proponemos la creación de espacios asociados a la web 3.0.

Como aportaciones presentamos la elaboración propuestas de valor asociadas al comercio móvil analizando tanto las características inherentes al canal, como los factores que según la literatura afectan al individuo a la hora de realizar compras a 
través de su dispositivo móvil. No conocemos ningún trabajo que haya realizado esta aproximación. Pensamos que proporcionamos con ellas una información relevante, tanto desde el punto de vista de la literatura como desde el punto de vista empresarial, ya que entendemos que una correcta combinación de factores de adopción- propuestas de valora facilitará el éxito empresarial. Como limitación del estudio debemos señalar que, pese a la revisión exhaustiva realizada de variables que ofrece la literatura, existe una dificultad de clasificación unívoca de las variables en cada grupo o tipo de factores. Por otra parte, es imposible incluir todos los trabajos existentes en un único estudio y hemos elegido los más representativos en cada caso según nos consta en nuestra revisión.

\section{Agradecimiento}

Esta investigación fue parcialmente subvencionada por el Ministerio de Economía y Competitividad (España) [número de referencia ECO2014-53060-R].

\section{REFERENCIAS}

AGREBI, S., y JALLAIS, J. (2015). "Explain the intention to use smartphones for mobile shopping", Journal of Retailing and Consumer Services, Vol. 22, p. $16-23$.

AJZEN, I. (1985). From intentions to actions: A theory of planned behaviour, en Kuhl J. y Beckman J. (Ed.), Action-control: From cognition to behaviour. Springer: Heidelberg, Alemania.

ANCKAR, B. y D'INCAU, D. (2002). "Value-added services in mobile commerce: An analytical framework and empirical findings from a national consumer survey". En System Sciences, HICSS. Proceedings of the 35th Annual Hawaii International Conference on IEEE, p. 1444-1453.

ANDERSON, J. C., NARUS, J. A. y VAN ROSSUM, W. (2006). "Customer Value propositions in business markets", Harvard Business Review, Vol. 84, $\mathrm{n}^{\circ} 3,91-99$.

AWASTHI, P., y SANGLE, P. S. (2012). "Adoption of CRM technology in multichannel environment: a review (2006-2010)", Business Process Management Journal, Vol. 18, $\mathrm{n}^{\circ}$ 3, p. 445-471.

BALASUBRAMAN, S., PETERSON, R. A. y JARVENPAA, S. L. (2002). "Exploring the Implications of M-Commerce for Markets and Marketing". Journal of the Academy of Marketing Science, Vol. 30, nº 4, p. 348-361.

BHATTI, T., (2007). "Exploring Factors Influencing the Adoption of Mobile Commerce". Journal of Internet Banking and Commerce, Vol. 12, n 3, p. 2 13. 
BIGNE, E., RUIZ, C. y SANZ, S. (2007). "Key drivers of mobile commerce adoption: an exploratory study of Spanish mobile users", Journal of Theoretical and Applied Electronic Commerce Research, Vol. 2, nº 2, p. 4860.

CHAN, F. T., y YEE-LOONG CHONG, A. (2013). "Analysis of the determinants of consumers'm-commerce usage activities", Online Information Review, Vol. $37, n^{\circ} 3$, p. 443-461.

CHEMINGUI, H., y BEN LALLOUNA, H. (2013). "Resistance, motivations, trust and intention to use mobile financial services", International Journal of Bank Marketing, Vol. 31, n ${ }^{\circ}$ 7, p. 574-592.

CHEN, L. (2008). "A model of consumer acceptance of mobile payment. International Journal of Mobile Communications, Vol. 6, $\mathrm{n}^{\mathrm{o}}$ 1, p. 32-52.

CLARKE, I. (2001). "Emerging value propositions for M-commerce", Journal of Business Strategies, Vol. 18, $\mathrm{n}^{\circ}$ 2, p. 133-149.

CONSTANTINESCU, M., ONUR, E., DURMUS, Y., NIKOU, S., DE REUVER, M., BOUWMAN, H., DJURICA, M. y GLATZ, P. (2014). "Mobile tethering: overview, perspectives and challengess", info, Vol. 16, no 3, p. 40-53.

DAVIS, F. D. (1989). "Perceived usefulness, Perceived Ease of Use, and User Acceptance of Information Technology", MIS Quaterly, Vol. 13, p. 319-339.

DAVIS, R., SAJTOS, L. y CHAUDHRI, A. A. (2011). "Do Consumers Trust Mobile Service Advertising?", Contemporary Management Research, Vol. 7, $\mathrm{n}^{\mathrm{o}} 4$, p. $245-269$.

FISHBEIN, M. y AJZEN, I. (1980). Understanding attitudes and predicting social behaviour. Prentice-Hall: New Jersey.

GROß, M. (2015). "Mobile shopping: a classification framework and literature review", International Journal of Retail and Distribution Management, Vol. $43, n^{\circ} 3$, p. 222-241.

GU, J., LEE, S. y SUH, Y. (2009). "Determinants of behavioural intention to mobile banking", Expert Systems with Applications, Vol. 36, p. 11605-11616.

HSU, C., LU, H. y HSU, H. (2007). "Adoption of the mobile Internet: An empirical study of multimedia message service (MMS)", Omega, Vol. 35, $\mathrm{n}^{\circ}$ 6, pp. 715-726.

HUNG, M.-C., YANG, S.-T. y HSIEH, T.-C. (2012), "An examination of the determinants of mobile shopping continuance", International Journal of Electronic Business Management, Vol. 10, no 1, p. 29-37.

ITU, UNIÓN INTERNACIONAL DE TELECOMUNICACIONES (2015). "Estadísticas". Disponible en: http://www.itu.int/en/ITUD/Statistics/Pages/stat/default.aspx (accedido el 30 de enero de 2015).

JAHANSHAHI, A. A., MIRZAIE, A., y ASADOLLAHI, A. (2011). "Mobile commerce beyond electronic commerce: Issue and challenges", Asian Journal of Business and Management Sciences, Vol. 1, n 2, p. 119-129. 
JAYAWARDHENA, C., KUCKERTZ, A., KARJALUOTO, H. y KAUTONEN, T. (2009). "Antecedents to permission based mobile marketing: an initial examination", European Journal of Marketing, Vol. 43, no 3/4, p. 473-499.

KANG, J. Y. M., y JOHNSON, K. K. (2013). "M-consumer segmentation: Mcommunication, M-distribution, and M-accessibility", International Journal of Marketing Studies, Vol. 5, nº 1, p. 86-95.

KATZ, E., BLUMLER, J. G. y GUREVICH, M. (1974). "Utilization of mass communication by the individual"., en Blumler, J. y Katz, E. (Eds.), The uses of mass communications: Current perspectives on gratifications research. Sage: Beverly Hills, CA.

KHALIFA, M. y SHEN, K. (2008). "Drivers for transactional B2C M-Commerce adoption: extended theory of planned behaviour". The journal of Computer Information Systems, Vol. 48, no 3, p. 111-117.

KHRAIM, H., YOUNES, E. AL S.I. y KHRAIM, A. S. (2011). "Factors Affecting Jordanian Consumers' Adoption of Mobile Banking Services", International Journal of Business and Social Science, Vol. 2, nº 20, p. 96-105.

KOIVUMÄKI, T., RISTOLA, A. y KESTI, M. (2008). "The effects of information quality of mobile information services on user satisfaction and service acceptance-empirical evidence from Finland", Behaviour and Information Technology, Vol. 27, $\mathrm{n}^{\circ}$ 5, p. 375-385.

LAUKKANEN, T. y PASANEN, M. (2008). "Mobile banking innovators and early adopters: How they differ from other online users?", Journal of Financial Services Marketing, Vol. 13, no 2, p. 86-94.

LE, C. y HO, J. C. (2010). "A Framework for Analyzing Business Model Innovation in Mobile Commerce", Journal of International Technology and Information Management, Vol. 19, $\mathrm{n}^{\circ}$ 4, p. 37-61.

LI, M., DONG, Z. Y., y CHEN, X. (2012). "Factors influencing consumption experience of mobile commerce: a study from experiential view", Internet Research, Vol. 22, no 2, p. 120-141.

LIÉBANA-CABANILLAS, J. F., SÁNCHEZ-FERNÁNDEZ, J., y MUÑOZLEIVA, F. (2014). "Role of gender on acceptance of mobile payment", Industrial Management and Data Systems, Vol. 114, $\mathrm{n}^{\circ}$ 2, p. 220-240.

LIU, F., ZHAO, X., CHAU, P. Y. y TANG, Q. (2015), "Roles of perceived value and individual differences in the acceptance of mobile coupon applications", Internet Research, Vol. 25, $\mathrm{n}^{\mathrm{0}}$ 3, p. 471-495.

LU, H. y SU, P. Y. (2009). "Factors affecting purchase intention on mobile shopping web sites", Internet Research, Vol. 19, n 4, p. 442-458.

LU, J. (2005). "Facilitating conditions, wireless trust and adoption intention", Journal of Computer Information Systems, Vol. 46, p. 17-24.

LU, J. (2014). "Are personal innovativeness and social influence critical to continue with mobile commerce?". Internet Research, Vol. 24, no 2, p. 134159. 
MALLAT, N., (2007). "Exploring consumer adoption of mobile payments. A qualitative study", The Journal of Strategic Information Systems, Vol. 16, $\mathrm{n}^{\circ}$ 4, p. 413-432.

MALLAT, N., ROSSI, M., TUUNAINEN, V. K. y ÖÖRNI, A. (2008). “An empirical investigation of mobile ticketing service adoption in public transportation", Personal and Ubiquitous Computing, Vol. 12, n ${ }^{\circ}$ 1, p. 57-65.

MAS, I. y RADCLIFFE, D. (2011). "Scaling mobile money", Journal of Payments Strategy and Systems, Vol. 5, n ${ }^{\circ}$ 3, p. 298-315.

NASRI, W., (2011). "Factors Influencing the Adoption of Internet Banking in Tunisia", International Journal of Business and Management, Vol. 6, $\mathrm{n}^{\circ}$ 8, $\mathrm{p}$. $143-160$.

ONTSI (2014). "B2C Electronic Commerce and The information society", Disponible en: http://www.ontsi.red.es (accedido el 29 de junio de 2015).

ONTSI (2015). "Indicadores", Disponible en: http://www.ontsi.red.es/ontsi/es/indicadores (accedido el 29 de junio de 2015).

PARK, J., SNELL, W., HA, S. y CHUNG, T. (2011). "Consumer's post-adoption of m-services: interest in future m-services based on consumer evaluations of current m-services", Journal of Electronic Commerce Research, Vol. 12, nº 3, p. $165-175$.

PEDERSEN, P. E., (2005). "Adoption of Mobile Internet Services: An Exploratory Study of Mobile Commerce Early Adopters", Journal of organizational computing and electronic commerce, Vol, 15, p. 203-222.

PORTER, M. E. (1985). Competitive Advantage - Creating and Sustaining Superior Performance, Free Press: New York.

PÜSCHEL, J., MAZZON, J. A. y HERNÁNDES, J. M. C. (2010). "Mobile banking: proposition of an integrated adoption intention framework", The International Journal of Bank Marketing, Vol. 28, $\mathrm{n}^{\circ}$ 5, p. 389-409.

RICHARD L, (1980). "A Cognitive Model of the Antecedents and Consequences of Satisfaction Decisions", Journal of Marketing Research, Vol. 17, $\mathrm{n}^{\mathrm{o}} 3$, p. 460-469

ROGERS, E. M., (1995). Diffusion of Innovations. $4^{\mathrm{a}}$ Ed. Free Press: Nueva York.

SONG, J., SAWANG, S., ANDREWS, L.Y. y DRENNAN, J. (2015). "Same but different? Mobile technology adoption in China", Information Technology \& People, Vol. 28, no 1, p. $107-132$.

SRIPALAWAT, J., THONGMAK, M.S. y NGRAMYARN, A. (2011). "Mbanking in metropolitan Bangkok and a comparison with other countries", The Journal of Computer Information Systems, Vol. 51, n 3, p. 67-76.

THAKUR, R., y SRIVASTAVA, M. (2013). "Customer usage intention of mobile commerce in India: an empirical study", Journal of Indian Business Research, Vol. $5, \mathrm{n}^{\mathrm{o}} 1$, p. 52-72.

THORBJØRNSEN, H., PEDERSEN, P. E. y NYSVEEN, H. (2009). "Categorizing networked services. The role of intrinsic-, user network- and complement 
network attributes", European Journal of Marketing, Vol. 43, n ${ }^{\circ}$ 3/4, p. 371397.

TURBAN, E., KING, D., LEE, J., WARKENTIN, M. y CHUNG, H. M. (2002). Electronic Commerce: A Managerial Perspective. Prentice Hall: Upper Saddle River, New Jersey.

TUREL, O., SERENKO, A. y BONTIS, N. (2007). "User acceptance of wireless short messaging services: Deconstruction perceived value", Information and Management, Vol. 44, $\mathrm{n}^{\mathrm{o}}$ 1, p. 63-73.

WU, J. y WANG, S. (2005). "What drives mobile commerce? An empirical evaluation of the revised technology acceptance model", Information and Management, Vol. 42, $\mathrm{n}^{\mathrm{o}}$ 5, p. 719-729.

WU, X., CHEN, Q., ZHOU, W. y GUO, J. (2010). "A review of Mobile Commerce consumers' behavior research: consumer acceptance, loyalty and continuance (2000-2009)", International Journal of Mobile Communications, Vol. 8, $\mathrm{n}^{\circ} 5$, p. 528-560.

YANG, Y., LIU, Y., LI, H., y YU, B. (2015). "Understanding perceived risks in mobile payment acceptance", Industrial Management and Data Systems, Vol. 115, n $^{\circ} 2$, p. $253-269$. .

YUAN, Y. y ZHANG, J.J. (2003). "Towards an appropriate business model for Mcommerce", International Journal of Mobile Communications, Vol. 1, $\mathrm{n}^{\mathrm{o}}$ $1 / 2$, p. 35-56.

YUN, H., LEE, C. C., KIM, B. G. y KETTINGER, W. J. (2011). "What Determines Actual Use of Mobile Web Browsing Services? A Contextual Study in Korea", Communications of the Association for Information Systems, Vol. 28, n 21, p. 313-328.

ZARMPOU, T., SAPRIKIS, V., MARKOS, A. y VLACHOPOULOU, M. (2012). "Modeling users' acceptance of mobile services", Electronic Commerce Research, Vol. 12, n⿳ 2 , p. 225-248.

ZHANG, R., CHEN, J. Q. y LEE, C. (2013). "Mobile commerce and consumer privacy concerns", Journal of Computer Information Systems, Vol. 53, $\mathrm{n}^{\mathrm{o}} 4$, p. 31-38.

ZHOU, T. (2014). "Understanding the determinants of mobile payment continuance usage”, Industrial Management y Data Systems, Vol. 114, nº 6, p. 936-948. 